\title{
Successful Treatment of Gastroparesis Following Total Esophagectomy Using Botulinum Toxin
}

A 52-year-old man with a malignant esophageal tumor underwent near-total esophagectomy with gastric replacement and feeding jejunostomy. Pyloroplasty was not performed. The postoperative course was unremarkable, except for excessive drainage from the nasogastric tube of $1000-1300 \mathrm{ml}$ per day from the first postoperative day.

Eight days later, a thick discharge appeared from the thoracic drain and 700$800 \mathrm{ml}$ was drained per day without any change in the patient's clinical condition. A barium meal demonstrated a leak from the cervical anastomosis, gastric dilatation with a large amount of fluid content, and very slow gastric emptying. Esophagogastroduodenoscopy revealed a $1-\mathrm{cm}$ hole $2 \mathrm{~cm}$ distal to the anastomosis, with a plastic drain emerging from it. The pylorus was dilated pneumatically with an 18-mm through-the-scope (TTS) balloon dilator in order to enhance gastric emptying. The drain was withdrawn by $2 \mathrm{~cm}$.

Ten days later, fluid discharge from the thoracic drain had ceased completely, but the nasogastric tube was still draining $600-1100 \mathrm{ml}$ per day. On the 37 th post- operative day, because there was no improvement in the delayed gastric emptying, $80 \mathrm{IU}$ of botulinum toxin (Botox; Allergan Pharmaceuticals, Westport, Co. Mayo, Ireland) was injected into the pylorus (20 IU into each quadrant). Three days after this procedure, drainage from the nasogastric tube ceased completely. A barium meal demonstrated normal evacuation of gastric content into the duodenum. Oral feeding was resumed gradually and the patient was discharged from hospital in a satisfactory condition. He remained asymptomatic during 6 months of follow-up.

After total esophagectomy with gastric cervical anastomosis the denervated thoracic stomach acts mainly as a tube conduit and empties by gravity alone [1]. It seems that the spastic pylorus delays effective gastric emptying and pyloric drainage procedures may be a solution. Resolution of this complication is usually spontaneous. In patients in whom resolution is delayed, the ideal would be to perform a minimally invasive procedure to bridge this delay until spontaneous improvement occurs. We decided to try the injection of botulinum toxin into the py- lorus, and this eventually proved successful. To the best of our knowledge, this is the first report of the use of botulinum toxin injection for the treatment of gastroparesis following esophagectomy.

\section{Competing interests: None}

A. Tcherniak', D. H. Kashtan², E. Melzer ${ }^{\mathbf{1}}$

${ }^{1}$ Department of Gastroenterology, Kaplan Medical Center, Rehovot, Israel

2 Department of Surgery B,

Kaplan Medical Center, Rehovot, Israel

\section{Reference}

${ }^{1}$ Velanovich V. Esophagogastrectomy without pyloroplasty. Dis Esophagus 2003; 16: $243-245$

\section{Corresponding Author}

\section{E. Melzer, M.D.}

Department of Gastroenterology Kaplan Medical Center

Rehovot 76100

Israel

Fax: $\quad+972-8-9413461$

E-mail: ehudm@clalit.org.il 